\title{
DETERMINAÇÃO DE POTÁSSIO EM ROCHAS SILICÁTICAS POR ESPECTROMETRIA DE EMISSÃO ATÔMICA COM CHAMA APÓS DISSOLUÇÃO ASSISTIDA POR ULTRASSOM
}

Daiane L. Baldez ${ }^{\mathrm{a}, \mathrm{b}}$, Liziane O. Avila ${ }^{\mathrm{a}}$, Daiane P. Torres ${ }^{\mathrm{a}}$, Rosane Martinazzo ${ }^{\mathrm{b}}$, Carlos A. P. Silveira ${ }^{\mathrm{b}}$ e Mariana A. Vieira ${ }^{\mathrm{a}, *}$

aLaboratório de Metrologia Química, Universidade Federal de Pelotas, 96010-900 Capão do Leão - RS, Brasil

bEmbrapa Clima Temperado, Rodovia BR 392, km 78, 96010-971 Pelotas - RS, Brasil

Recebido em 06/04/2018; aceito em 02/08/2018; publicado na web em 19/09/2018

\begin{abstract}
DETERMINATION OF POTASSIUM IN SILICATE ROCKS BY FLAME ATOMIC EMISSION SPECTROMETRY AFTER ULTRASOUND DISSOLUTION. This work presents the development of an analytical method for sample preparation aiming at the $\mathrm{K}$ determination in silicate rocks (basalt, monzogranite and shale fines) following ultrasound dissolution and analysis by F AES. Sample preparation method was optimized by using fractional factorial designs. The following optimal conditions were obtained for each silicate rock: $0.1 \mathrm{~g}$ of sample mass for any of the three samples, and volumes of $\mathrm{HNO}_{3}, \mathrm{HCl}$ and $\mathrm{HF}$, respectively, of $2.5,0.8$ and $2.5 \mathrm{~mL}$ for basalt; $3.5,0.8$ and $2.5 \mathrm{~mL}$ for monzogranite, and $2.5,1.0$ and $2.0 \mathrm{~mL}$ for shale fines. Sample dissolution was achieved by using the ultrasound bath at room temperature $\left(25^{\circ} \mathrm{C}\right)$ for 1 hour. The accuracy was evaluated through the analysis of two certified reference materials, for which there was no statistical difference between the certified and the found $\mathrm{K}$ concentration values $(95 \%$ confidence level). The $\mathrm{K}$ concentrations determined were: $1.45 \pm 0.01$ for basalt; $4.19 \pm 0.03$ for monzogranite and $1.60 \pm 0.02$ for shale fines. The developed method presented accurate and reliable results for $\mathrm{K}$ amounts in silicate rocks and is simple and safe, which are important features for the implementation of a methodology as a routine analysis.
\end{abstract}

Keywords: potassium; silicate rocks; ultrasound dissolution; F AES.

\section{INTRODUÇÃo}

O Brasil tem avançado em termos de produtividade, eficiência e sustentabilidade em diversos sistemas de produção agrícolas, porém ainda apresenta alta dependência de importação de insumos, especialmente matérias-primas para fertilizantes que em sua grande maioria, se apresentam na forma de sais solúveis, a saber, fontes de nitrogênio, potássio e fósforo, os quais são essenciais para o desenvolvimento de qualquer cultura agrícola.,

A busca por sistemas agrícolas sustentáveis, com baixo uso de insumos externos, diversificados e eficientes em termos energéticos, representa grande preocupação dos pesquisadores, agricultores e formuladores de políticas agrícolas em todo o mundo., Uma alternativa para a diminuição do uso de fertilizantes de elevada solubilidade é o uso de rochas moídas para a produção agrícola, também conhecido como remineralização de solos. Esta é uma técnica acessível, ecologicamente correta e consiste no princípio da liberação de nutrientes, que compõem os minerais da rocha, de forma gradual para o solo. Devido a menor solubilidade em comparação aos fertilizantes solúveis, o emprego de rochas moídas, apresenta efeito residual por vários ciclos agrícolas, fornecendo macro e micro nutrientes para as plantas e promovendo também, para determinadas rochas, o aumento da capacidade de troca de cátions, em razão da formação de novos minerais de argila durante o processo de intemperismo dos minerais. ${ }^{1,5-8}$

Os fertilizantes potássicos mais utilizados na agricultura, encontram-se na forma de sais de potássio como o $\mathrm{KCl}, \mathrm{o} \mathrm{K}_{2} \mathrm{SO}_{4}, \mathrm{o} \mathrm{K}_{2} \mathrm{SO}_{4}$. $\mathrm{MgSO}_{4}$ e o $\mathrm{KNO}_{3}$. Considerando que aproximadamente $90 \%$ do $\mathrm{K}$ utilizado como fertilizante no Brasil é importado, é oportuno considerar fontes não convencionais seguras do mesmo e, principalmente fontes que apresentem baixo custo. Neste cenário, pesquisas vêm sendo realizadas sobre o possível aproveitamento de rochas silicáticas para produção e/ou aplicação direta como fontes de K, bem como de

*e-mail: marianavieira@pq.cnpq.br outros nutrientes na tentativa de reduzir a lixiviação de nutrientes e, também, diminuir a carência de fertilizante e a dependência externa nacional de insumos agrícolas. ${ }^{2-5}$ Estas rochas podem ser aplicadas no solo na forma pura, em misturas ou após serem submetidas a tratamentos térmicos ou químicos. . $^{1,3,5,6}$

Com foco na regulamentação dos pós de rochas, no dia 10 de dezembro de 2013 foi promulgada, pela Presidência da República, a Lei Federal $n^{\circ} 12.890 / 2013^{9}$ incluindo-os como uma categoria de insumo destinado à agricultura, denominada remineralizadores. Em 5 de 10 de março de 2016, o Ministério da Agricultura, Pecuária e Abastecimento (MAPA) publicou a Instrução Normativa (IN) $\mathrm{n}^{\circ} 5,{ }^{10}$ que regulamenta e define critérios para o uso de remineralizadores e condicionadores de solo estabelecendo as especificações e garantias mínimas desses produtos. Dessa forma, estudos voltados para o desenvolvimento de métodos de análises para a caracterização de remineralizadores de solo são de suma importância para dar suporte ao registro e uso adequado destes insumos na agricultura.

Entretanto, rochas são amostras complexas de difícil decomposição e o procedimento mais indicado para essas matrizes é a utilização de ácidos concentrados em combinação com aquecimento em vasos abertos ou em sistemas fechados, podendo ser empregado tanto aquecimento condutivo quanto a decomposição assistida por radiação micro-ondas. ${ }^{11}$

Diferentes métodos de preparo de amostras de rochas e materiais similares têm sido reportados. O método US EPA 3052 $\mathrm{a}^{12}$ permite a dissolução completa de amostras silicáticas e se enquadra bem com os critérios de análise elementar em materiais geológicos.

Nos últimos anos, houve um acentuado desenvolvimento de métodos que utilizam as ondas ultrassônicas para o preparo de diferentes amostras, para posterior determinação de elementos por técnicas de espectrometria atômica. ${ }^{13,14}$ Krug e Rocha ${ }^{11}$ enfatizaram em uma recente publicação, as características do emprego das ondas ultrassônicas e os cuidados que devem ser tomados durante o preparo de amostras usando estes métodos. Estudos empregando o ultrassom no preparo de amostras podem ser encontrados na literatura: Perin et al. ${ }^{15}$ 
utilizaram o ultrassom para extrair As e Se em amostras de solos submetidos à remineralizadores; Oreste et al.${ }^{16}$ determinaram $\mathrm{Cu}, \mathrm{Fe}$, $\mathrm{Mg}$ e Zn em louças cerâmicas de uso doméstico através da extração assistida por ultrassom com $\mathrm{HNO}_{3} \mathrm{e} \mathrm{HF}$; Timm et al. ${ }^{17}$ empregaram a extração assistida por ultrassom para a determinação de $\mathrm{Cu}, \mathrm{Cd}, \mathrm{Pb}$ e Cr em sedimentos do Canal São Gonçalo em Pelotas/RS; Leite et al. ${ }^{18}$ utilizaram a extração assistida por ultrassom para a determinação de $\mathrm{As}, \mathrm{Cd}, \mathrm{Cr}, \mathrm{Pb}$ e Se em fertilizantes orgânicos. Esses estudos reforçam o poder da utilização deste tipo de preparo de amostra, tendo em vista, sobretudo, tempo e custos reduzidos.

Com base no exposto, o objetivo deste trabalho foi desenvolver um método analítico simples, robusto e rápido para a determinação de $\mathrm{K}$ em rochas silicáticas por espectrometria de emissão atômica com chama (F AES), preparadas utilizando a dissolução assistida por ultrassom.

\section{PARTE EXPERIMENTAL}

\section{Instrumentação}

As determinações de $\mathrm{K}$ foram realizadas em modo emissão, utilizando um espectrômetro de absorção/emissão atômica modelo AAnalyst 200 (Perkin Elmer, Connecticut, EUA), com chama de ar/acetileno, nas vazões de $10,0 \mathrm{~L} \mathrm{~min}^{-1}$ e $2,5 \mathrm{~L} \mathrm{~min}^{-1}$, respectivamente; comprimento de onda de $766,49 \mathrm{~nm}$ e fenda espectral de $2,7 \mathrm{~mm}$, conforme as recomendações do fabricante.

Para o preparo das amostras foi utilizado um banho ultrassônico, modelo Elmasonic S $40(\mathrm{H})$, com potência nominal de $140 \mathrm{~W}$ e frequência ultrassônica de 37 kHz (Elma, Singen, Alemanha). As amostras foram pesadas utilizando uma balança analítica Ohaus Adventurer, modelo AR 2140 (Pine Brook, NJ, EUA), com uma resolução de 0,1 mg e tara máxima de $210 \mathrm{~g}$.

\section{Reagentes e amostras}

Todos os reagentes utilizados neste estudo foram de grau analítico. Para o preparo das soluções foi utilizada água deionizada, obtida a partir de um sistema de destilação em vidro, modelo MA078/5 (Marconi, Brasil) e, posteriormente, eluída em um desionizador modelo CS1800 Evolution (Permution, Brasil). Uma solução estoque contendo $10000 \mathrm{mg} \mathrm{L}^{-1}$ de K (Fluka, Buchs, Alemanha) foi utilizada.

Para a dissolução das amostras de rochas foi utilizado $\mathrm{HNO}_{3}$ $65 \%(\mathrm{~m} / \mathrm{m})$ (Vetec, Brasil), bidestilado abaixo do seu ponto de ebulição no destilador de quartzo MA 075 (Marconi, Brasil). Nessa etapa também foi utilizado ácido HF $40 \%(\mathrm{~m} / \mathrm{m})$ (Vetec, Brasil), $\mathrm{HCl} 38 \%(\mathrm{~m} / \mathrm{m})$ (Sigma-Aldrich, Brasil) e $\mathrm{H}_{3} \mathrm{BO}_{3} 99,5 \%(\mathrm{~m} / \mathrm{m})$ (Sigma-Aldrich, EUA).

Para avaliar a exatidão, os seguintes materiais de referência certificados foram utilizados: CRM MBL-1 (Basalto) e CRM MGL-1 OShBO (Granito), provenientes do Central Geological Laboratory (CGL, Mongólia).

As amostras de rochas como fonte de $\mathrm{K}$ investigadas foram o basalto, o monzogranito e finos de xisto, todas cedidas pela Embrapa Clima Temperado (Pelotas/RS), as quais fazem parte de um banco de amostras constituído por diversas rochas coletadas em diferentes locais da Região Sul do Brasil. A amostra de basalto é proveniente da Pedreira Carolo, em Montenegro (RS); a de monzogranito da Pedreira Silveira, em Pelotas (RS) e a amostra de finos de xisto é proveniente da Petrobras/SIX, em São Mateus do Sul (PR). Todas as amostras foram adequadas quanto às suas granulometrias de modo que $100 \%$ de cada material apresentou partículas menores que $0,3 \mathrm{~mm}$.

\section{Otimização do método de preparo de amostra}

Os parâmetros experimentais investigados para o desenvolvimento do método tiveram como base o descrito pela US EPA $3052 \mathrm{~A}^{12}$ que orienta que a decomposição total de matrizes complexas contendo silicatos deve ser feita a partir de um tratamento ácido e abertura em forno de micro-ondas.

Um planejamento fatorial fracionário $2^{5-2}$ foi utilizado para a otimização do método de preparo das amostras das diferentes rochas silicáticas investigadas, usando o programa STATISTICA (STATSOFT STATISTICA 7.0). Para tal, foram utilizadas as seguintes variáveis experimentais e respectivos níveis: massa da amostra (100 e $200 \mathrm{mg})$, volumes de $\mathrm{HNO}_{3}(2,5$ e $3,0 \mathrm{~mL}), \mathrm{HCl}(0,8$ e $1,0 \mathrm{~mL})$ e $\mathrm{HF}$ (2,5 e 3,0 mL) e tempo de sonicação (60 e 120 minutos). Os ensaios foram realizados de forma aleatória, de acordo com a ordem dos experimentos que foi gerada pelo software.

A Tabela 1S (MS) apresenta o planejamento fatorial aplicado. Este primeiro planejamento resultou em condições ótimas para as amostras basalto e monzogranito, conforme discussão posterior. Para a amostra de finos de xisto foi necessário aplicar um novo planejamento fatorial fracionário do tipo $2^{4-1}$, objetivando uma combinação mais adequada das condições experimentais (Tabela 2S). Neste, os seguintes fatores foram avaliados, também em dois níveis: massa da amostra, e volumes de $\mathrm{HNO}_{3}, \mathrm{HCl}$ e $\mathrm{HF}$.

De acordo com as condições ótimas para cada amostra, estas foram pesadas em triplicata, diretamente em frascos volumétricos de polipropileno (PP). Após, fez-se a adição dos ácidos $\mathrm{HNO}_{3}, \mathrm{HCl}$ e HF, e os frascos foram transferidos para o banho ultrassônico. As amostras foram sonificadas à temperatura ambiente, com os frascos semiabertos, para possível alívio da pressão. Após 1 hora de ultrassom, as mesmas foram aferidas a $50 \mathrm{~mL}$ com água desionizada. $\mathrm{O}$ aspecto final das soluções é apresentado na Figura 1. Além dessa diluição inicial das amostras para $50 \mathrm{~mL}$, foi necessária uma segunda diluição para adequação à faixa linear da curva de calibração. Para basalto uma alíquota de $0,2 \mathrm{~mL}$ foi diluída para um volume final de $10 \mathrm{~mL}$; monzogranito: $0,1 \mathrm{~mL}$ para $20 \mathrm{~mL}$; e finos de xisto: $0,1 \mathrm{~mL}$ para $10 \mathrm{~mL}$.

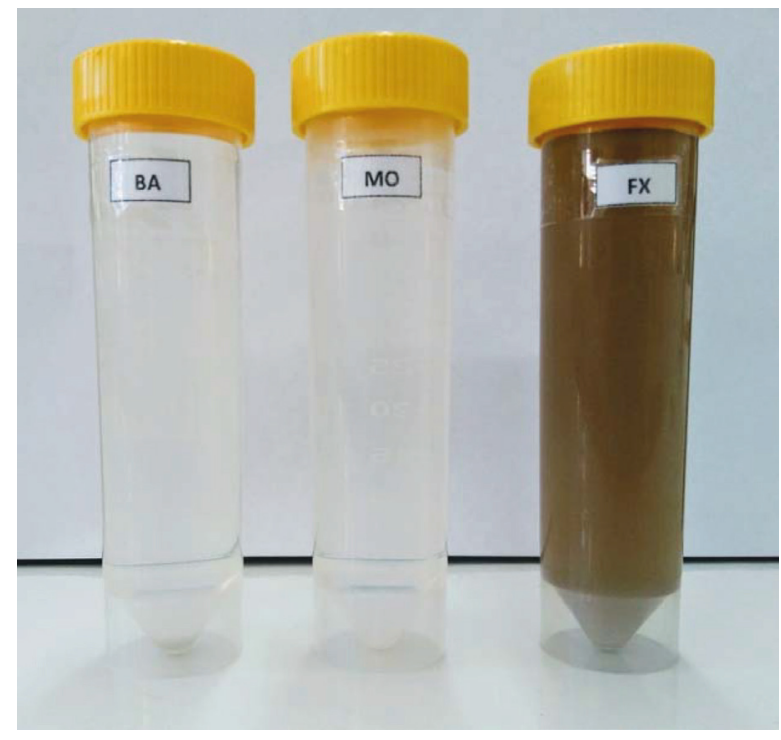

Figura 1. Amostras de basalto (BA), monzogranito (MO) e finos de xisto (FX) após dissolução por ultrassom

Como pode ser observado, as amostras de basalto e de monzogranito obtiveram uma completa dissolução, apresentando soluções límpidas e transparentes, diferentemente da amostra de finos de xisto que apresentou coloração marrom e material em suspensão. 
Essa coloração é uma característica natural da rocha em estudo, por se tratar de folhelho carbonoso sedimentar, apresentando entre seus constituintes um componente orgânico, o querogênio ${ }^{19}$ Embora a amostra visualmente não tenha ficado límpida em comparação com as outras, não foi constatado problemas na determinação da concentração de K, uma vez que a sua exatidão foi comprovada por teste de adição e recuperação em vários níveis de concentração de $\mathrm{K}$.

Uma solução de $\mathrm{H}_{3} \mathrm{BO}_{3} 4,5 \%$ (m/v) foi adicionada às amostras em uma segunda etapa de diluição, previamente à leitura, a fim de complexar o HF remanescente11 e evitar que o mesmo danificasse partes do nebulizador no espectrômetro. A quantidade necessária de $\mathrm{H}_{3} \mathrm{BO}_{3}$ foi calculada seguindo a estequiometria da reação, na qual para cada $1 \mathrm{~mL}$ de $\mathrm{HF} 40 \%(\mathrm{~m} / \mathrm{m})$, foram necessários $8 \mathrm{~mL}$ da solução $4,5 \%$ (m/v) de $\mathrm{H}_{3} \mathrm{BO}_{3}$ para a reação entre o $\mathrm{HF}$ e o $\mathrm{H}_{3} \mathrm{BO}_{3}$. Os brancos analíticos foram preparados de maneira similar às amostras e as curvas de calibração foram construídas em meio aquoso.

Para verificar a exatidão do método, foram utilizados dois materiais de referência certificados e também o teste de adição e recuperação em vários níveis de concentração do K. Foram estabelecidos os níveis de adição em termos percentuais de $\mathrm{K}$ nas rochas investigadas (a saber, menores que o teor de $\mathrm{K}$ para monzogranito, de cerca de $4,44 \%$ ), sendo nível 1 de $25 \%, 2$ de $50 \%$ e 3 de $75 \%$.

\section{RESULTADOS E DISCUSSÃO}

\section{Otimização do método de preparo de amostra}

Utilizando o planejamento fatorial fracionário $2^{5-2}$ foram obtidos os gráficos normais dos efeitos para a determinação de K nas amostras de rochas. O gráfico normal de efeito padronizado é uma alternativa para avaliar os resultados de um planejamento fatorial, completo ou fracionário, a fim de avaliar os efeitos dos fatores experimentais investigados. ${ }^{20} \mathrm{~A}$ Figura $2 \mathrm{~A}$ apresenta o gráfico normal de efeitos para o planejamento fatorial fracionário $2^{5-2}$ para a amostra de basalto.
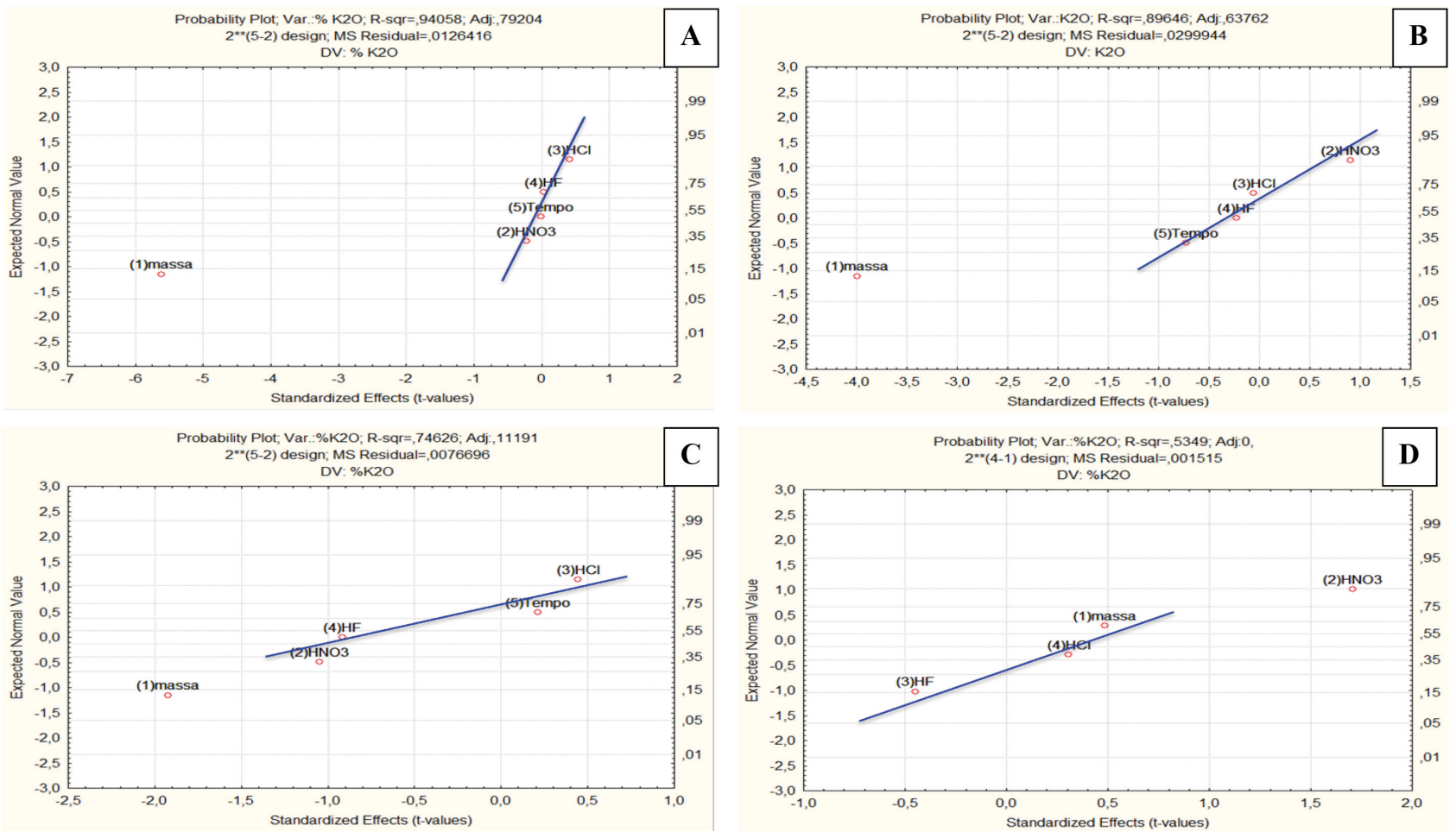

Figura 2. Gráfico dos valores dos efeitos padronizados para o planejamento fatorial fracionário $2^{5-2}$ para (A) basalto; (B) monzogranito e (C) finos de xisto. Variáveis: massa de amostra, $\mathrm{HNO}_{3}, \mathrm{HCl}, \mathrm{HF}$ e tempo de sonicação.(D) Planejamento fatorial fracionário $2^{4-1}$ para os finos de xisto. Variáveis: massa de amostra, $\mathrm{HNO}_{3}, \mathrm{HCl}$ e $\mathrm{HF}$.

É possível observar que os valores dos efeitos dos três ácidos e do tempo de sonicação se ajustam bem à linha reta, a qual está centralizada próximo dos zeros dos eixos das ordenadas e das abscissas. Pode-se então inferir que estes são efeitos não significativos, derivados unicamente de flutuações experimentais, e assim se referem a erros aleatórios. ${ }^{20,21}$ Segundo Torres, ${ }^{22}$ na situação em que os efeitos dos fatores avaliados se ajustam bem a uma linha reta que cruza o valor normal igual à zero (ou probabilidade acumulada de 50\%) sobre o ponto zero do eixo horizontal, faz sentido considerar que esses pontos sejam provenientes de uma população normal de média zero. Deste modo, os níveis testados para os ácidos $\mathrm{HNO}_{3}, \mathrm{HCl}$ e $\mathrm{HF}$, assim como o tempo de sonicação para a dissolução do basalto, não impactaram significativamente a resposta analítica e podem ser usados de acordo com a conveniência analítica, ou seja, dentro dos níveis testados pode-se escolher as menores quantidades dos ácidos e menos tempo de sonicação, sem nenhum prejuízo para a confiabilidade dos resultados das determinações do teor de $\mathrm{K}$ medido nas amostras de basalto submetidas a essas condições testadas.

Por outro lado, o efeito da massa de amostra foi muito marcante e discrepante do comportamento dos efeitos dos outros fatores avaliados, não sendo considerado um erro aleatório e sim um efeito significativo. Ao variar a massa de 100 para $200 \mathrm{mg}$, houve um efeito significativo e negativo na resposta analítica, indicando que uma maior quantidade de massa de amostra prejudica a recuperação do $\mathrm{K}$ nas condições avaliadas para a dissolução. Isso está relacionado ao fato de que as rochas silicáticas são matrizes de difícil solubilização, e com o aumento da massa de amostra a ser digerida, é necessário também aumentar a quantidade dos ácidos empregados. Sendo assim, optou-se pela utilização de uma massa de $100 \mathrm{mg}$ de modo a obter uma dissolução mais eficiente das rochas, com maior recuperação do analito. Portanto, mantendo-se o fator massa de amostra fixo em 100 $\mathrm{mg}$, o método para a dissolução ácida em ultrassom de basalto para a determinação de $\mathrm{K}$ por F AES, avaliado via planejamento fatorial fracionário $2^{5-2}$, alcançou as condições ótimas para todos os fatores

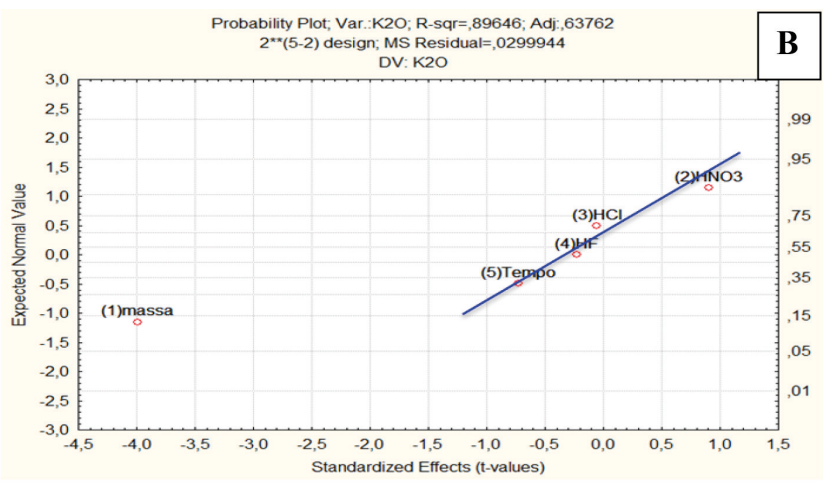


investigados, e assim, optou-se por utilizar o menor nível de volumes de ácidos e também de tempo de sonicação.

A Figura 2B apresenta o gráfico normal dos efeitos do planejamento fatorial fracionário $2^{5-2}$ para a amostra de monzogranito. Dentre as variáveis testadas no experimento, os valores dos efeitos dos ácidos $\mathrm{HCl}$, $\mathrm{HF}$ e $\mathrm{HNO}_{3}$, e do tempo de sonicação, ficaram próximos da linha reta, mais ou menos centralizada nos zeros do eixo das ordenadas e das abscissas, podendo, portanto, ser considerados como provenientes de erros aleatórios, conforme discutido anteriormente. Assim como para a matriz de basalto, a massa de amostra também teve um efeito significativo na resposta de $\mathrm{K}$ para o monzogranito, o qual se encontra bem distante da linha reta que ajusta bem os outros parâmetros experimentais, não podendo o efeito da massa para o monzogranito ser considerado como proveniente apenas de erros aleatórios. Ao variar a massa de 100 para $200 \mathrm{mg}$, houve um efeito na resposta analítica, evidenciando que maior massa de amostra diminuiu a recuperação do K para monzogranito. Desta maneira, optou-se também pela utilização da menor quantidade de massa de amostra para uma melhor dissolução das amostras.

Como os dois níveis avaliados dos fatores $\mathrm{HCl}(0,8$ e $1 \mathrm{~mL}), \mathrm{HF}$ (2,5 e $3 \mathrm{~mL}), \mathrm{HNO}_{3}(2,5$ e 3,0 mL) e tempo de sonicação (60 e 120 minutos) não impactaram significativamente a resposta analítica de $\mathrm{K}$ para o monzogranito, optou-se pela utilização dos menores volumes de ácido, exceto o $\mathrm{HNO}_{3}$, para o qual, apenas por precaução, optou-se por usar 3,0 mL, considerando que seu efeito parece estar no limiar da significância (efeito menos ajustado na linha reta do gráfico de probabilidade). Nestas condições e com a massa de amostra fixada em $100 \mathrm{mg}$, pode-se considerar que o método para a dissolução ácida assistida por ultrassom e determinação por F AES foi completamente otimizado também para monzogranito.

Para a rocha finos de xisto, o gráfico normal de efeitos apresentado na Figura 2C mostra que, entre as variáveis investigadas, a massa teve um efeito significativo e foi o fator avaliado mais crítico, tendo em vista o valor negativo alto do seu efeito padronizado, e a distância do ajuste da reta para o efeito dos outros fatores avaliados. Em contrapartida, os ácidos $\mathrm{HCl}, \mathrm{HNO}_{3}$ e $\mathrm{HF}$ apresentaram valores de efeito padronizado no limiar do que pode ser considerado somente erro aleatório, os quais de fato podem prejudicar a eficiência do processo de dissolução. O tempo de sonicação, entretanto, teve seu efeito padronizado localizado bem próximo ao zero dos eixos horizontal e vertical (ou 50\% de probabilidade acumulada), e, portanto, pode ser considerado não significativo.

Assim, visando o alcance da otimização completa e confiável da dissolução da rocha finos de xisto para a determinação de K por F AES, os parâmetros massa de amostra, $\mathrm{HCl}, \mathrm{HF}$ e $\mathrm{HNO}_{3}$ foram submetidos a um planejamento experimental de refinamento, um planejamento fatorial fracionário $2^{4-1}$, a fim de verificar os níveis ótimos a ser empregados dos ácidos e massa de amostra.

Destaca-se aqui que o banho ultrassônico empregado opera com programação de no máximo 30 minutos, e antes do início da etapa seguinte de mais 30 minutos de exposição ao ultrassom, as amostras ainda não apresentavam aspecto de dissolução total da matriz. Desta forma, para garantir a dissolução bem-sucedida da amostra, decidiu-se empregar no mínimo 60 minutos de ultrassom para todas as amostras, considerando a complexidade de matrizes rochosas, sendo que a eficiência de dissolução das mesmas foi estatisticamente idêntica a 60 ou 120 minutos de exposição ao ultrassom, como supracitado.

No segundo planejamento experimental para a amostra de finos de xisto, os níveis avaliados para os fatores foram: massa da amostra
(100 e $125 \mathrm{mg}), \mathrm{HNO}_{3}$ e $\mathrm{HF}(2,0$ e $2,5 \mathrm{~mL})$ e $\mathrm{HCl}(1,0$ e $1,2 \mathrm{~mL})$. A Figura 2D mostra o gráfico normal de efeitos para o planejamento fatorial fracionário $2^{4-1}$.

Avaliando o gráfico dos efeitos padronizados $2 \mathrm{D}, \mathrm{o} \mathrm{HCl}$ (níveis 1,0 e $1,2 \mathrm{~mL}$ ), a massa de amostra (níveis 100 e $125 \mathrm{mg}$ ) e o HF (níveis 2,0 e 2,5 mL) não apresentaram efeitos significativos para os novos níveis avaliados, implicando assim em valores de recuperação de $\mathrm{K}$ estatisticamente iguais para a amostra finos de xisto. Assim, optou-se por adotar os menores níveis desses fatores como a condição ótima. Finalmente, o volume de $\mathrm{HNO}_{3}$ foi o fator mais crítico do planejamento de refinamento do finos de xisto, cujo valor do efeito padronizado não pôde ser ajustado à linha reta do gráfico dos outros efeitos padronizados, sendo assim um fator estatisticamente significativo. Portanto, a redução de $\mathrm{HNO}_{3}$ de 2,5 $\mathrm{mL}$ para $2,0 \mathrm{~mL}$ impactou negativamente a dissolução da amostra, e o nível de $2,5 \mathrm{~mL}$ deve ser mantido para uma melhor dissolução e valores de recuperação de $\mathrm{K}$ mais próximos de $100 \%$.

Em resumo, em todos os casos, com as três diferentes rochas investigadas, a massa de amostra sempre foi um parâmetro com pouca robustez, em que para maiores massas de amostra, os valores de recuperação de $\mathrm{K}$ foram mais baixos. Levando em consideração a homogeneidade de amostras sólidas investigadas no presente trabalho, podemos considerá-las como misturas heterogêneas. As rochas, os solos e os sedimentos são materiais que em geral apresentam composições heterogêneas. Massas menores que 100 mg tendem a comprometer a representatividade e homogeneidade de amostra complexas. ${ }^{11} \mathrm{~A}$ Tabela 1 apresenta um resumo das condições ótimas obtidas após aplicação dos planejamentos fatoriais fracionários.

Tabela 1. Resumo das condições ótimas, após aplicação dos planejamentos fatoriais fracionários, para determinação de $\mathrm{K}$ em rochas silicáticas por F AES

\begin{tabular}{lccc}
\hline Fatores & Basalto & Monzogranito & Finos de Xisto \\
\hline Massa (mg) & 100 & 100 & 100 \\
$\mathrm{HNO}_{3}(\mathrm{~mL})$ & 2,5 & 3,0 & 2,5 \\
$\mathrm{HCl}(\mathrm{mL})$ & 0,8 & 0,8 & 1,0 \\
$\mathrm{HF}(\mathrm{mL})$ & 2,5 & 2,5 & 2,0 \\
Tempo de sonicação (min) & 60 & 60 & 60 \\
\hline
\end{tabular}

\section{Parâmetros de mérito}

Os parâmetros de mérito para a determinação de $\mathrm{K}$ nas amostras foram obtidos a partir dos dados da curva de calibração. Na Tabela 2 pode-se observar que a faixa linear de trabalho utilizada é adequada para o método proposto. A curva de calibração foi construída em meio aquoso e apresentou adequada linearidade com ajuste satisfatório, observado através do coeficiente de determinação, bem como sensibilidade adequada para a quantificação. Os limites de detecção e quantificação alcançados se apresentaram satisfatórios para a determinação de $\mathrm{K}$ nas amostras de rochas silicáticas investigadas.

\section{Avaliação da exatidão}

Com o intuito de avaliar a exatidão do método para a determinação de $\mathrm{K}$ em rochas silicáticas pela técnica de F AES, dois materiais de referência certificados de origem geológica foram utilizados. Os

Tabela 2. Parâmetros de mérito para a determinação de K por F AES em rochas silicáticas após dissolução ácida assistida por ultrassom

\begin{tabular}{ccccc}
\hline Analito & Faixa Linear $\left(\mathrm{mg} \mathrm{L}^{-1}\right)$ & $\mathrm{a}\left(\mathrm{L} \mathrm{mg}^{-1}\right)$ & $\mathrm{R}^{2}$ & $\mathrm{LD}^{\left(\mathrm{mg} \mathrm{L} \mathrm{L}^{-1}\right)}$ \\
\hline $\mathrm{K}$ & $0,1-1,2$ & 19672,8 & 0,997 & 0,002 \\
\hline
\end{tabular}

a: coeficiente angular da curva; LD: limite de detecção; LQ: limite de quantificação; R²: coeficiente de determinação. 
mesmos foram preparados de acordo com o método desenvolvido e os resultados obtidos são apresentados na Tabela 3.

Tabela 3. Teor de $\mathrm{K}_{2} \mathrm{O}$ e intervalo de confiança, expressos em $\% \mathrm{~K}_{2} \mathrm{O}$, obtidos para CRMs, pelo método de dissolução ácida assistida por ultrassom e determinação de K por F AES ( $\mathrm{n}=3$ )

\begin{tabular}{lcc}
\hline & \multicolumn{2}{c}{$\% \mathrm{~K}_{2} \mathrm{O} \pm \mathrm{IC}^{*}$} \\
\cline { 2 - 3 } & $\begin{array}{c}\text { Valor } \\
\text { Certificado }\end{array}$ & $\begin{array}{c}\text { Valor } \\
\text { Encontrado }\end{array}$ \\
\hline CRM MBL-1 (Basalto) & $3,90 \pm 0,04$ & $4,03 \pm 0,55$ \\
CRM MGL-1 OShBO (Granito) & $3,58 \pm 0,04$ & $3,34 \pm 0,28$ \\
\hline
\end{tabular}

*IC: Intervalo de confiança: $\alpha=0,05$.

De acordo com os resultados apresentados na Tabela 3, foi possível atestar a concordância entre os resultados obtidos e os valores certificados para o analito em estudo com $95 \%$ de confiança, o que comprova a exatidão do método desenvolvido. Os resultados encontrados não apresentaram diferenças significativas em relação aos valores certificados no nível de confiança calculado. Com base nos valores relativamente baixos dos desvios padrão relativos (RSDs), menores que 5\%, a alta precisão do método proposto também pode ser comprovada.

O teste de adição e recuperação do analito também foi aplicado, visando assim avaliar a exatidão (via \% de recuperação do analito) e a precisão (via desvio padrão) do método desenvolvido, bem como os possíveis efeitos de matriz. Os resultados obtidos são apresentados na Tabela 4.

Tabela 4. Níveis de concentração adicionado e recuperados para K (\%) nas amostras de basalto, monzogranito e finos de xisto após dissolução ácida assistida por ultrassom e determinação de K por F AES ( $n=3$ )

\begin{tabular}{lccc}
\hline Amostra & $\begin{array}{c}\text { Adicionado } \\
(\% \mathrm{~K})^{*}\end{array}$ & $\begin{array}{c}\text { Encontrado } \\
(\% \mathrm{~K} \pm \mathrm{SD})\end{array}$ & $\begin{array}{c}\text { Recuperação } \\
(\%)\end{array}$ \\
\hline \multirow{3}{*}{ Basalto } & 0 & $1,40 \pm 0,05$ & - \\
& 0,36 & $1,70 \pm 0,01$ & 83,3 \\
& 0,73 & $2,02 \pm 0,01$ & 84,9 \\
Monzogranito & 1,09 & $2,35 \pm 0,05$ & 87,2 \\
\hline \multirow{3}{*}{ Finos de Xisto } & 0 & $4,44 \pm 0,35$ & - \\
& 1,29 & $5,56 \pm 0,28$ & 86,8 \\
& 2,58 & $6,92 \pm 0,03$ & 96,1 \\
& 3,23 & $7,65 \pm 0,21$ & 99,4 \\
\hline
\end{tabular}

SD: Desvio padrão. * \% de $\mathrm{K}$ adicionado em relação ao teor de $\mathrm{K}$ original na rocha.

De acordo com os resultados apresentados na Tabela 4, pode-se verificar uma faixa de recuperação adequada para os níveis de adição avaliados de $\mathrm{K}$, variando entre 83,3 a $110,6 \%$. Constatou-se ainda que o método apresenta exatidão adequada para análise proposta. Além disso, foram encontrados baixos valores de desvio padrão, o que evidencia também uma alta precisão.

\section{Determinação de $\mathrm{K}$ nas amostras de rochas}

Os teores de $\mathrm{K}$ obtidas nas três amostras das rochas silicáticas investigadas, preparadas usando o método de dissolução assistida por ultrassom, são apresentados na Tabela 5.
Tabela 5. Teores de $\mathrm{K}$ e $\mathrm{K}_{2} \mathrm{O}$ (\%) obtidos nas amostras de basalto, monzogranito e finos de xisto por F AES $(\mathrm{n}=3)$

\begin{tabular}{lccc}
\hline Amostra & $\mathrm{K}(\%)$ & $\mathrm{K}_{2} \mathrm{O}(\%)$ & $\mathrm{RSD}(\%)$ \\
\hline Basalto & 1,45 & 1,77 & 0,39 \\
Monzogranito & 4,19 & 5,02 & 0,54 \\
Finos de Xisto & 1,60 & 1,90 & 1,11 \\
\hline
\end{tabular}

RSD: desvio padrão relativo.

\section{CONCLUSÃO}

O método de dissolução ácida assistida por ultrassom se mostrou adequado e eficiente para a determinação de $\mathrm{K}$ em rochas silicáticas por F AES, já que possibilitou a recuperação quantitativa do analito nas três amostras de rochas investigadas, em condições ambientes de temperatura e pressão, além do emprego de baixas quantidades de ácidos, tempo de sonicação relativamente curto e segurança operacional.

Os resultados obtidos apresentaram alta exatidão, o que foi atestado pelas satisfatórias recuperações dos materiais de referência certificados analisados e pelos baixos valores obtidos de desvio padrão relativo, assim como teste de adição e recuperação em três níveis do analito. Desta forma, o método pode ser considerado exato, confiável, rápido e simples para o preparo de amostras de rochas visando a determinação de $\mathrm{K}$ por $\mathrm{F}$ AES.

\section{MATERIAL SUPLEMENTAR}

No material suplementar, disponível gratuitamente em http:// quimicanova.sbq.org.br na forma de arquivo PDF, estão apresentados na Tabela $1 \mathrm{~S}$, os dados para o planejamento fatorial e na Tabela $2 \mathrm{~S}$, o novo planejamento fatorial realizado para a amostra de finos de xisto.

\section{AGRADECIMENTOS}

Os autores agradecem à Fundação de Amparo à Pesquisa do Estado do Rio Grande do Sul (FAPERGS) - Programa Pesquisador Gaúcho (Processo 2027-2551/13-3 SIAFEM) pelo apoio financeiro e à Embrapa Clima Temperado e Petrobrás-SIX pela bolsa de Mestrado de D. L. Baldez. M. A. Vieira agradece ao Conselho Nacional de Desenvolvimento Científico e Tecnológico (CNPq) pelo apoio financeiro através do Edital Universal (Processo no 447552/2014-7) e pela Bolsa de Produtividade em Pesquisa Nível 2 (Processo $n^{\circ}$ 310917/2013-1).

\section{REFERÊNCIAS}

1. Pillon, C. N.; Anais do III Congresso Brasileiro de Rochagem, Pelotas, Brasil, 2017. Embrapa Clima Temperado. p. 15.

2. Teixeira, A. M. S.; Sampaio, J. A.; Garrido, F. M. S.; Medeiros, M. E.; Holos 2012, 5, 21.

3. Penha, M. N. C.; Dissertação de Mestrado, Universidade Federal de São Carlos, Brasil, 2016.

4. Silva, D. J.; Faria, C. M. B.; Mendes, A. M. S.; Morais, A. T.; Anais da Reunião Brasileira de Manejo e Conservação do Solo e da Água, Aracaju, Brasil, 2006.

5. Cola, G. P. A.; Simão, J. B. P.; Revista Verde 2012, 7, 1.

6. Theodoro, S. H.; Leonardos, O.; Rocha, E. L.; Rego, K. G.; Espaço \& Geografia 2006, 9, 263.

7. ANDA - Associação Nacional para a Difusão de Adubos. Anuário estatístico. 175 p.; 2015.

8. Silva, D. R. G.; Marchi, G.; Spehar, C. R.; Guilherme, L. R. G.; Rein, T. A.; Soares, D. A.; Ávila, F. W.; Rev. Bras. de Ciênc. Solo, 2012, 36, 951. 
9. Presidência da República casa civil. Lei $\mathrm{n}^{\circ} 12890$, de 10 de dezembro de 2013. Inclui os remineralizadores como uma categoria de insumo destinado à agricultura, e dá outras providências. Brasília, 2013.

10. Ministério da Agricultura, Pecuária e Abastecimento (MAPA). Instrução Normativa $\mathrm{n}^{\circ} 53$, de 23 de outubro de 2013. http://www.agricultura.gov. br/assuntos/insumos-agropecuarios/insumos-agricolas/fertilizantes/ legislacao/in-53-2013-com-as-alteracoes-da-in-6-de-10-3-16.pdf

11. Krug, F.J.; Rocha, F. R. P.; Métodos de preparo de amostras para análise elementar, 1 ed., EDIT-SBQ, São Paulo, 2016.

12. EPA. Method 3052. Microwave assisted acid digestion of siliceous and organically based matrices, 1996. <http://www.epa.gov/osw/hazard/ testmethods/sw846/online/3_series.htm> Acessada em maio de 2018.

13. Carvalho, L. R. F.; Souza, S. R.; Martinis, B. S.; Korn, M.; Anal. Chim. Acta, 1995, 317, 171.

14. Korn, M.; Andrade, M. V. A. S.; Borges, S. S.; Sousa, C. S.; Oliveira, F. S.; J. Braz. Chem. Soc. 2003, 14, 254.

15. Perin, E. C.; Torres, D. P.; Becker, S.; Rombaldi, C. V.; Silveira, C. A. P.; Anais do III Congresso Brasileiro de Rochagem, Pelotas, Brasil, 2017. Embrapa Clima Temperado. p. 45.
16. Oreste, E. Q.; De Souza, A. O.; Pereira, C. C.; Vieira, M. A.; Ribeiro, A. S.; Quím. Nova 2017, 40, 310

17. Timm, J. G.; Pinto, A. M. T. P.; Alves, M. M.; Clasen, C. D. Filho, P. J. S.; Ribeiro, A. S.; Vieira, M. A.; Ecotoxicol. Environ. Contam., no prelo.

18. Leite, D. P.; Tese de Doutorado - Universidade Federal de São Paulo, Brasil, 2015.

19. Ribas, L.; Neto, J. M. R.; França, A. B.; Alegre, H. K. P.; J. Pet. Sci. Eng. 2017, 152, 156

20. Barros Neto, B.; Scarminio, I. S.; Bruns, R. E. (2007). Como fazer experimentos: Pesquisa e desenvolvimento na ciência e na indústria ( $3^{\mathrm{a}}$ edição ed.). Campinas, SP, Brasil: Editora da UNICAMP.

21. Torres, D. P.; Martins-Teixeira, M. B.; Silva, E. F.; Queiroz, H. M.; Food Addit. Contam.; 2012, 29, 625 .

22. Torres, D. P.; Tese de Doutorado, Universidade Estadual de Campinas, 2013. 